\title{
COMPARISON OF ANTIFUNGAL EFFECTS OF COMMERCIALLY AVAILABLE HERBAL MOUTHWASHES AND CHLORHEXIDINE AGAINST CANDIDA ALBICANS IN DIABETIC PATIENTS: AN IN VITRO STUDY
}

\author{
CHANDINI RAVIKUMAR ${ }^{1 *}$, RADHIKA ARJUNKUMAR ${ }^{2}$, GOPINATH PRAKASAM ${ }^{3}$ \\ ${ }^{1}$ Department of Periodontics, Saveetha Dental College, Vellapanchavadi, Chennai, Tamil Nadu, India. ${ }^{2}$ Department of Periodontics, \\ Saveetha Dental College, Vellapanchavadi, Chennai, Tamil Nadu, India. ${ }^{3}$ Department of Microbiology, Saveetha Dental College, \\ Vellapanchavadi, Chennai, Tamil Nadu, India. Email: chandiniravikumar14@gmail.com
}

Received: 04 June 2016, Revised and Accepted: 08 June 2016

\section{ABSTRACT}

Objective: Candida albicans is a part of the normal flora of the mouth in diabetes mellitus (DM) patients. Periodontitis is one of the main complications in diabetic patients. Mechanical and chemical plaque control are the most productive methods in preventing periodontal diseases in the oral cavity. The objective of this study is to compare the in vitro effect of herbal mouthwashes and chlorhexidine (CHX) against C. albicans.

Methods: Saliva samples were obtained from diabetic patients reporting for treatment to Saveetha medical college. C. albicans was cultured from the salivary sample. A yeast suspension was made by sub culturing the C. albicans. The mouthwashes used in the study are HiOra regular (0.2\%), HiOra sensitive $(0.5 \%$ ), and clohex plus (CHX gluconate $0.02 \%$ ) mouthwashes. The fungal suspension was spread on Sabouraud's dextrose agar (SDA) plates with a sterile swab. Subsequently, wells of $6 \mathrm{~mm}$ in diameter were made with a suitable distance using sterile cork borer on pre-inoculated agar plates and filled with $100 \mu \mathrm{l}$ of each mouthwashes. From the zones of inhibition seen, antimicrobial activity was expressed in terms of average diameter of the zones of inhibition measured.

Results: Using HiOra regular mouthwash, 13/18 (72\%) wells were found to show zone of inhibition $\geq 20 \mathrm{~mm}$. In HiOra sensitive mouthwash, only $9 / 18$ (50\%) showed inhibition zone $\geq 20 \mathrm{~mm}$. With effect of regular CHX mouthwash, none of the strains showed the zone of inhibition to be $\geq 20 \mathrm{~mm}$. Most of the strains responded well with all the three mouthwashes.

Conclusion: Among the 2 herbal mouthwashes, HiOra regular mouthwash was most effective in inhibiting the candidal growth when compared to the HiOra sensitive. HiOra regular mouthwash still showed better inhibitory actions when compared to the regular CHX mouthwash and the candidal species showed increased sensitivity to it.

Keywords: Candida albicans, Chlorhexidine mouthwash, Diabetes mellitus, Herbal mouthwashes, Zone of inhibition.

(C) 2016 The Authors. Published by Innovare Academic Sciences Pvt Ltd. This is an open access article under the CC BY license (http://creativecommons. org/licenses/by/4. 0/) DOI: http://dx.doi.org/10.22159/ajpcr.2016.v9i5.13275

\section{INTRODUCTION}

It is believed that dental plaque is the main etiological factor that causes caries, gingivitis, and periodontal disease [1]. Mechanical and chemical plaque control are the most productive methods in preventing periodontal diseases in the oral cavity. Various chemical agents have been advocated for the prevention of dental plaque, which are available in the form of a mouthwash [2]. The mechanical supra gingival plaque control includes the use of toothbrush, floss, wood sticks, and interdental brushes. Since the effective use of these oral hygiene products may be beyond the ability of majority of the patients, a chemical plaque control approach, is desirable to deal with the potential deficiencies of daily self-performed oral hygiene. Therefore, plaque control represents the cornerstone of good oral hygiene practice [3]. Chlorhexidine (CHX) is regarded as the "gold standard" anti plaque agent. Since it is prone to cause certain adverse effects, there is a need for a naturally occurring indigenous and cost-effective oral hygiene aid, such as herbal mouthwashes. CHX is also widely used as an antimicrobial agent and medicament in periodontal treatments [4]. HiOra (himalaya herbals) mouthwash has antiplaque, analgesic, antimicrobial, antiseptic, and refreshing properties. It protects the teeth against common strains of bacteria, which cause periodontitis leading to sensitive teeth. It also helps in restoring the mineral composition of the teeth and helps in maintaining optimum health of the gums and teeth [5]. The constituents of HiOra mouthwash are Salvadora persica, Terminalia bellerica, Piper betle, Gossia fragrantissima, Elettaria cardamomum, Mentha spp., and Trachyspermum. It has been highly effective against candidal species [6]. Candida albicans is considered as part of the normal flora of the mouth. Using mouthwashes is a common way to control $C$. albicans population in the mouth, which are used widely in dentistry [7]. Mouthwashes have been recommended for the prevention and control of oral diseases, and among the antifungal therapies, fluconazole is the first-line of management option for oral infections caused by Candida species [8]. Periodontitis, a chronic inflammatory disease, is four times more common in persons with diabetes mellitus (DM) and is considered to be the sixth most common complication of DM. Inversely, persistent poor glycemic control has been associated with a greater incidence and progression of gingivitis and periodontitis [9]. Diabetics, particularly insulin-dependent DM patients, are considered as immunocompromised, though it is not easy to clearly characterise the immunologic deficiencies [10]. In earlier studies, the antiplaque effects of herbal mouthwashes have been proven. However, their antifungal efficacy was not reported in the previous literature studies. Henceforth, it is essential to compare the antifungal efficacy of herbal mouthwashes and CHX for a better understanding, that when taking into account, the side-effects of CHX presently tested herbal mouthwash may be considered as a good alternative [11].

\section{METHODS}

Unstimulated salivary samples were collected from diabetic patients who reported to Saveetha Medical College, Thandalam. Their glycosylated hemoglobin (HbA1c) levels and blood glucose levels were noted to confirm their diabetic status. Ethical Committee approval was obtained to proceed with the research study. 


\section{Isolation of $C$ albicans}

In this study, a sum of 20 clinical strains of $C$. albicans were isolated from diabetic patients (Fig. 1). All strains were cultivated in chrome agar, and placed at $30^{\circ} \mathrm{C}$ for $24 \mathrm{hrs}$ until activated. From the 20 saliva samples of diabetic patients, 2 were excluded in the study as the fungal growth was found to be nil in these subjects and 18 clinical strains of C. albicans was included.

\section{Mouthwashes}

The mouthwashes used in the study are HiOra regular $(0.2 \%)$, HiOra sensitive $(0.5 \%)$, and clohex plus (0.2\%) (CHX gluconate) mouthwashes.

\section{Antimicrobial screening by well diffusion method}

A yeast suspension was made by sub culturing $C$. albicans culture into normal saline and the OD that matched to a McFarland standard of 0.5 in which it was assumed that the number of cells were $1.5 \times 108 \mathrm{CFU} / \mathrm{ml}$. The fungal suspension was spread on Sabouraud's dextrose agar plates with a sterile swab. Subsequently, wells of $6 \mathrm{~mm}$ in diameter were made with suitable distance using sterile cork borer on pre-inoculated agar plates and filled with $100 \mu \mathrm{l}$ of each mouthwashes then allowed to diffuse at room temperature for 2 hrs for proper diffusion. The presence of zone of inhibition was regarded as the presence of antimicrobial action. From the inhibition zones seen, antimicrobial activity was expressed in terms of average diameter of the zones of inhibition measured [12].

\section{RESULTS}

In this study, we have attempted to check the effectiveness of 2 herbal mouthwashes, HiOra regular, and HiOra sensitive mouthwash against 18 clinical strains of $C$. albicans isolated from diabetic patients using well diffusion method. All these strains were also subjected to the regular CHX mouthwash, clohex plus, as a control group.

Using HiOra regular mouthwash, 13/18 (72\%) were found to show zone of inhibition greater than or equal to $20 \mathrm{~mm}$. Fig. 2 shows the maximum zone of inhibition of $30 \mathrm{~mm}$ exhibited by HiOra regular mouthwash. In HiOra sensitive mouthwash, only $9 / 18$ (50\%) showed inhibition zone $\geq 20 \mathrm{~mm}$. With effect of regular CHX mouthwash, none of the strains showed zone of inhibition to be greater than or equal to $20 \mathrm{~mm}$.

Certain strains showed the least and no zone of inhibition when compared to the others (Table 1). However, most of the strains responded well with all the three mouthwashes.

The mean number and standard deviation values of all the three mouthwashes are shown (Table 2). HiOra regular mouthwash showed the highest mean and standard deviation values.

The zones of inhibition between the two herbal mouthwashes, (HiOra regular and HiOra sensitive), of CHX and between all the three mouthwashes are compared by using post hoc tests (Table 3 ).

There is a statistically significant difference in the zone of inhibition by the HiOra regular and HiOra sensitive mouthwashes when compared to CHX mouthwash. However, there is no statistically significant difference between the zone of inhibition produced by HiOra regular and HiOra sensitive mouthwashes.

\section{DISCUSSION}

From the results, it is evident that the herbal mouthwashes showed a higher value of zone of inhibition when compared to CHX, which is considered to be the gold-standard mouthwash. HiOra regular mouthwash, which showed increased efficacy against the growth of C. albicans, is proved to be a good alternative to CHX for its antimicrobial, antiplaque, analgesic, and refreshing properties.

Severe periodontitis at baseline was associated with an increased risk of poor glycemic control (HbA1c $>9.0 \%$ ) at follow-up (minimum 2 years), suggesting that severe periodontitis was a risk factor

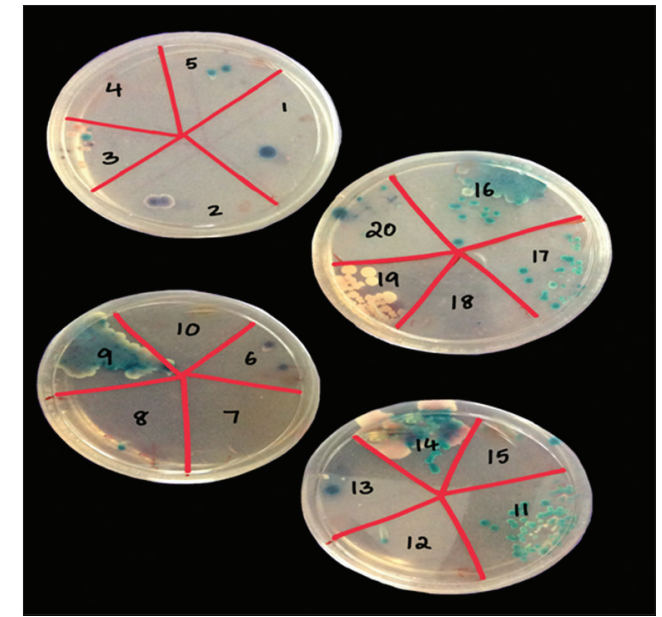

Fig. 1: The candida isolates from 20 diabetic patients. Number 4 and 10 were excluded for showing nil growth

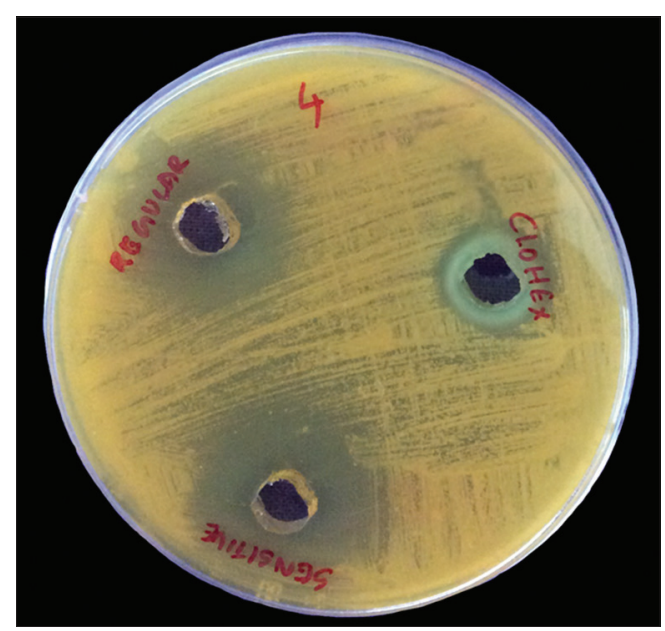

Fig. 2: HiOra regular and HiOra sensitive mouthwashes shows maximum zone of inhibition of $30 \mathrm{~mm}$ and $23 \mathrm{~mm}$, respectively Chlorhexidine shows the least value of inhibition zone of only $15 \mathrm{~mm}$

for compromised diabetes management [13]. Therefore, while conducting the study, the HbA1c levels and blood glucose levels of the diabetic patients were collected. Though monitoring the glycemic status in these diabetic patients was not our primary concern, the relationship between these levels and candidal carriage had to be determined for a clearer understanding. It was found that in most of them, their Hba1c levels and blood glucose levels were more than the normal range [14].

Substantial evidence has been demonstrating DM as a risk factor for the impairment of periodontal health and a growing body of evidence has been supporting Parkinson's disease as having an adverse effect on glycemic control and on the pathophysiology of diabetes-related complications. Hence, it becomes mandatory to control the periodontal status in DM patients with poor glycemic control [15].

In earlier studies, HiOra herbal mouthwash has shown a good potential as an anti-plaque agent in comparison to CHX gluconate mouthwash and has proven to be equally effective. HiOra Herbal mouthwash due to its natural ingredients has no reported side-effects and can serve as a good alternative to patients who wish to avoid alcohol (e.g., xerostomia), sugar (e.g., diabetics), any artificial preservatives, and artificial colors in their mouth rinses [16]. 
Table 1: Values of zone of inhibition by 3 mouthwashes included in the study

\begin{tabular}{llll}
\hline S. No & $\begin{array}{l}\text { Zone of } \\
\text { inhibition by } \\
\text { HiOra regular } \\
\text { mouthwash }\end{array}$ & $\begin{array}{l}\text { Zone of } \\
\text { inhibition by } \\
\text { HiOra sensitive } \\
\text { mouthwash }\end{array}$ & $\begin{array}{l}\text { Zone of } \\
\text { inhibition } \\
\text { by clohex } \\
\text { mouthwash }\end{array}$ \\
\hline 1 & Nil & Nil & 13 \\
2 & 21 & 17 & 14 \\
3 & 22 & 20 & 17 \\
4 & 30 & 23 & 15 \\
5 & 24 & 20 & 18 \\
6 & Nil & Nil & 12 \\
7 & 25 & 18 & 13 \\
8 & 20 & 17 & 15 \\
9 & 27 & 20 & 16 \\
10 & 22 & 18 & 17 \\
11 & 21 & 19 & 17 \\
12 & 17 & 21 & 15 \\
13 & 26 & 20 & 11 \\
14 & 20 & 22 & 17 \\
15 & 25 & 27 & 17 \\
16 & Nil & Nil & 15 \\
17 & 16 & 13 & 18 \\
18 & 28 & 29 & \\
\hline
\end{tabular}

Table 2: Zone of inhibition

\begin{tabular}{ll}
\hline Mouthwashes & Mean \pm SD \\
\hline HiOra regular mouthwash & $22.93 \pm 3.973$ \\
HiOra sensitive mouthwash & $20.27 \pm 3.955$ \\
Clohex plus mouthwash & $15.17 \pm 2.121$
\end{tabular}

*The units of mean and standard deviation of inhibition zone are in millimeters $(\mathrm{mm})$

Table 3: Post hoc tests (multiple comparisons)

Dependent variable: Zone of inhibition Bonferroni

\begin{tabular}{llc}
\hline (I) Group & (J) group & Significance \\
\hline HiOra regular & HiOra sensitive mouthwash & 0.109 \\
mouthwash & Clohex plus mouthwash & 0.000 \\
HiOra sensitive & HiOra regular mouthwash & 0.109 \\
mouthwash & Clohex plus mouthwash & 0.000 \\
Clohex plus & HiOra regular mouthwash & 0.000 \\
mouthwash & HiOra sensitive mouthwash & 0.000 \\
\hline
\end{tabular}

\section{CONCLUSION}

From the multiple research studies and a few systematic reviews, it gives us a conclusion that herbal mouthwashes could be used as a definite alternative to $\mathrm{CHX}$ on a daily basis to maintain oral hygiene and also to treat plaque-induced gingival diseases as effectively as CHX. In various studies conducted earlier, herbal mouthwashes were proved to be excellent antiplaque agents. In the present study, it is concluded that the herbal mouthwashes are equally good antifungal agents and HiOra regular mouthwash was most effective in inhibiting the candidal growth. It showed better inhibitory actions when compared to the regular CHX mouthwash and the candidal species showed increased sensitivity to it.

\section{REFERENCES}

1. Shetty PR, Setty SB, Kamat SS, Aldarti AS, Shetty SN. Comparison of the anti gingivitis and anti plaque efficacy of the herboral (herbal extract) mouthwash with chlorhexidine and listerine mouthwashes: A clinical study. Pak Oral Dent J 2013;33(1):76-81.

2. Biswas S. Comparative evaluation of the effect of green tea, listerine and chlorhexidine mouth washes in gingivitis patients: A randomized controlled trial. Sch J Dent Sci (SJDS) 2015;2(1):104-12.

3. Bagchi S, Saha S, Jagannath GV, Reddy VK, Sinha P. Evaluation of efficacy of a commercially available herbal mouthwash on dental plaque and gingivitis: A double-blinded parallel randomized controlled trial. J Indian Assoc Public Health Dent 2015;13(3):222-7.

4. Prasad KA, John S, Deepika V, Dwijendra KS, Reddy BR, Chincholi S. Anti-plaque efficacy of herbal and $0.2 \%$ chlorhexidine gluconate mouthwash: A comparative study. J Int Oral Health 2015;7(8):98-102.

5. Kaur RK, Singh MP, Chopra R, Bhatia A. Evaluation of efficacy of three commercially available herbal mouthwashes in treatment of chronic gingivitis: A comparative clinical study. Int J Dent Med Res 2014;1(4):42-6

6. Jangid K, Doraiswamy JN, Saji VS, Malaiappan S. Efficacy of herbal mouthwashes compared to chlorhexidine in gingivitis - A systematic review. Int J Ethnobiol Ethnomed 2014;1(1):1-12.

7. Talebi S, Sabokbar A, Riazipour M, Saffari M. Comparison of the in vitro effect of chemical and herbal mouthwashes on Candida albicans. Jundishapur J Microbiol 2014;7(12):e12563.

8. Martin MV. The use of fluconazole and itraconazole in the treatment of Candida albicans infections: A review. J Antimicrob Chemother 1999;44(4):429-37.

9. Casqueiro J, Casqueiro J, Alves C. Infections in patients with diabetes mellitus: A review of pathogenesis. Indian J Endocrinol Metab 2012;16 Suppl 1:S27-36.

10. Prasad CE. Immunodeficiencies in diabetes and mycobacterial infections. Int J Diabetes Dev Ctries 1999;19:52-5.

11. Bhati N, Mitra R, Reddy JJ, Oza S, Vinayak KM. Evaluation of efficacy of chlorhexidine and a herbal mouthwash on dental plaque: An in vitro comparative study. Int J Pharm Bio Sci 2013;4(3):(B)625-32.

12. Jeevitha $\mathrm{T}$, Deepa $\mathrm{K}$, Michael A. In vitro evaluation of antimicrobial activity of chemical and Aloe vera mouthwashes against Candida albicans. World J Pharm Res 2015;4(8):1829-38.

13. Preshaw PM, Alba AL, Herrera D, Jepsen S, Konstantinidis A, Makrilakis K, et al. Periodontitis and diabetes: A two-way relationship. Diabetologia 2012;55(1):21-31.

14. Kaur S, Pallagatti S, Sheikh S, Singh VA, Gupta R, Gupta D. Salivary glucose levels, oral candidal carriage, candidal strain diversity and antifungal susceptibility of candida among adult diabetic patients. Dent Oral Craniofac Res 2016;2(1):206-11.

15. Negrato CA, Tarzia O, Jovanovic L, Chinellato LE. Periodontal disease and diabetes mellitus. J Appl Oral Sci 2013;21(1):1-12.

16. Chitnis PJ, Dadlani HP, Parwani SR, Parwani RN, Prasad SV Comparative evaluation of anti-plaque efficacy of herbal and $0.2 \%$ chlorhexidine gluconate mouthwash in a 4-day plaque re-growth study. J Indian Soc Periodontol 2013;17(1):72-7. 